\title{
Analyses of test beam data using the CALICE calorimeter
}

\author{
Mikhail Danilov ${ }^{1 *}$ \\ Institute for Theoretical and Experimental Physics \\ B.Cheremushkinskaja 25, 117218 Moscow, Russia \\ E-mail: daniloveitep.ru
}

Abstract

We discuss several features of hadronic showers recorded in the high granularity CALICE Si-W ECAL and scintillator-tile HCAL and confront them with several Geant4 physics models. The first measurements of the time-structure of hadronic showers in a tungsten scintillator calorimeter are presented. The high granularity of the CALICE calorimeters allows a software compensation which improves the energy resolution by $\sim 20 \%$ in the $10-80 \mathrm{GeV}$ energy range. The first experimental tests of the Particle Flow Algorithm (PFA) have been performed using overlaid pion showers. The PandoraPFA demonstrated excellent performance with real data.

The 2011 Europhysics Conference on High Energy Physics, EPS-HEP2011

Grenoble, Rhône-Alpes, France

July 21-27 2011

$1 \quad$ Speaker

* On behalf of the CALICE Collaboration 


\section{Introduction}

Detectors for a future $\mathrm{e}^{+} \mathrm{e}^{-}$Linear Collider (ILC or CLIC) plan to use a Particle Flow Algorithm (PFA) to achieve unprecedented energy resolution for jets. Using PFAs, ideally only the energy of neutral particles is measured in the calorimeters, while the charged particle energy is reconstructed in a tracker where the resolution is much better. The PFA needs a high granularity calorimeter capable to separate showers created by charged and neutral particles.

The CALICE collaboration has constructed and studied several high granularity prototypes of calorimeters for future liner colliders. In this paper we discuss several features of hadronic showers recorded in the CALICE Si-W electromagnetic calorimeter (ECAL)[1] and iron scintillator-tile hadron calorimeter (AHCAL)[2]. The ECAL and AHCAL collected data at CERN and FNAL in 2006-2010. In 2010 the AHCAL iron absorber was substituted by a tungsten absorber which is planned to be used in a CLIC detector.

\section{Comparison between measured shower properties and MC calculations}

The test beam setup was simulated with Mokka(v7.02)[3], a Geant4 based Monte Carlo (MC) application. Various Geant4 physics lists have been used for the comparison with the experimental data[4,5]. Events have been simulated with Geant 4.9.3.

The lateral shower profile determines to a large extent the performance of the PFA approach. The weighted mean shower radius measured in the AHCAL[6] is 5-10\% larger than MC predictions. MC calculations with different physics lists reproduce well the central part of the shower but underestimate the tail by $10-30 \%$. The initial part of pion showers was studied using the ECAL[4]. Most physics lists underestimate the mean shower radius by about $10 \%$.

The high granularity of the CALICE calorimeters makes it possible to identify a point where the incident hadron makes its first interaction. The shower longitudinal profile measured from this point is much narrower than the profile measured from the front of the calorimeter. This makes comparison with MC models much more sensitive. The longitudinal shower profile in the ECAL is shown in Fig.1[4]. It is compared with simulations using different physics lists. The particle composition of the shower is quite different in these models and none of them describe data well. The largest differences between models and data are observed at the first calorimeter layers. Disagreement between the data and models is somewhat reduced at higher pion energies. The FTFP_BERT physics list, while not perfect, gives the best overall description of the longitudinal shower development in the ECAL. The FTFP_BERT_TRV physics list gives the best (although not perfect) description of the longitudinal shower profile in the AHCAL for pion energies from 10 to $80 \mathrm{GeV}$ [5].

The CLIC detector needs time stamping of events. Therefore knowledge of the time structure of hadron showers is very important. To provide the first measurements of the shower time structure in the tungsten-scintillator calorimeter, T3B, a special timing experiment[7] was installed after the last layer of the tungsten HCAL. The mean time of the first hit in T3B tiles has been measured at different radial distances from the shower axis. Simulations with the QGSP_BERT physics list overshoot the data strongly. However, the variant with high precision neutron tracking, QGSP_BERT_HP, describes the data very well. 

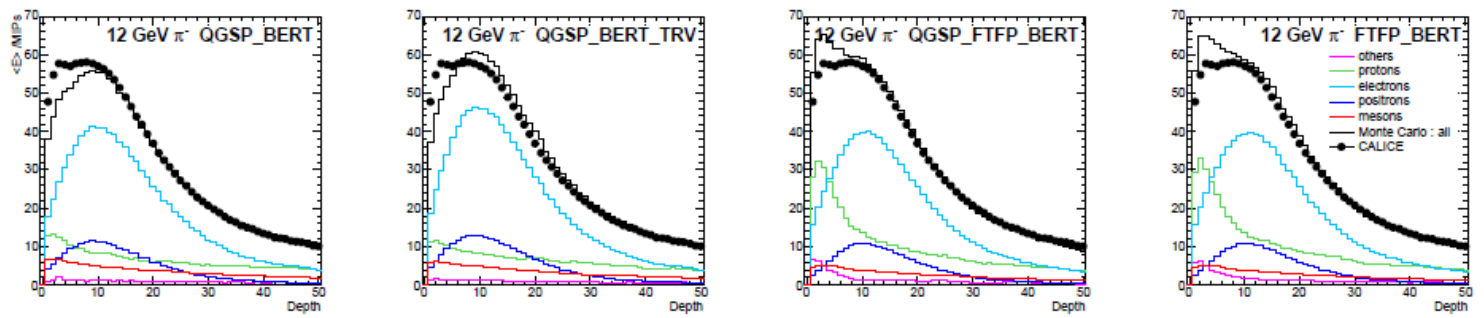

Figure 1: Longitudinal energy profiles for $12 \mathrm{GeV} \pi$ - data (shown as points), compared with simulations using different physics lists. The mean energy in MIPs is plotted against the depth after the initial interaction, in units of effective $1.4 \mathrm{~mm}$ tungsten layers. The breakdown of the Monte Carlo into the energy deposited by different particle categories is also indicated.

\section{Software compensation and PFA tests}

The AHCAL is a non-compensating calorimeter. It has different response to electrons and hadrons of the same energy. This leads to a deterioration of the energy resolution since the electromagnetic fraction of hadronic showers has large fluctuations. The AHCAL fine granularity allows to estimate the electromagnetic fraction for each event and to improve considerably the energy resolution. Two software compensation approaches have been developed[8]. In the local approach each hit is weighted according to its energy. The weights are determined from the data sub sample not used in the further analysis. In the global approach the shower energy of each event is multiplied by just one factor which depends on the fraction of hits with high energy in this shower (see Fig.2, left). The local and global approaches give almost identical improvement in the energy resolution of about $20 \%$ in the $10-80 \mathrm{GeV}$ energy range (see Fig.2, right) with linearity better than $2 \%$.

Linear collider detectors are optimized using the PFA approach. The PFA programs were developed and tuned using only MC simulations. The first experimental test of the PFA was performed using overlaid pion showers detected in the CALICE calorimeters[9]. The incoming

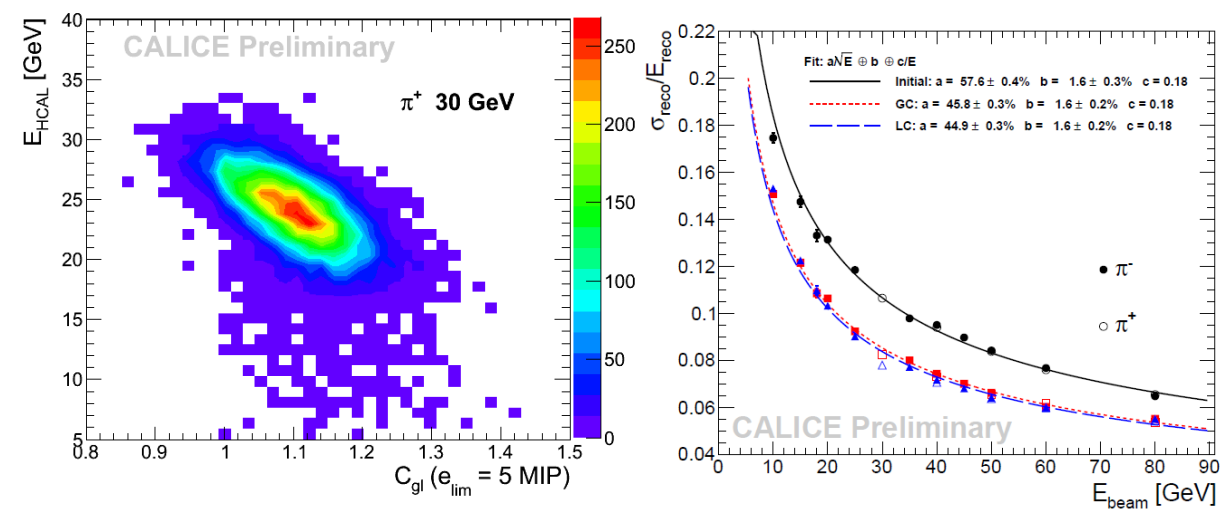

Figure 2: Correlation between measured energy and the global correction factor (left).Relative resolution versus beam energy without compensation (black circles and solid line) and after local (LC - blue triangles and dashed line) and global (GC - red squares and dotted line) compensation (right) 

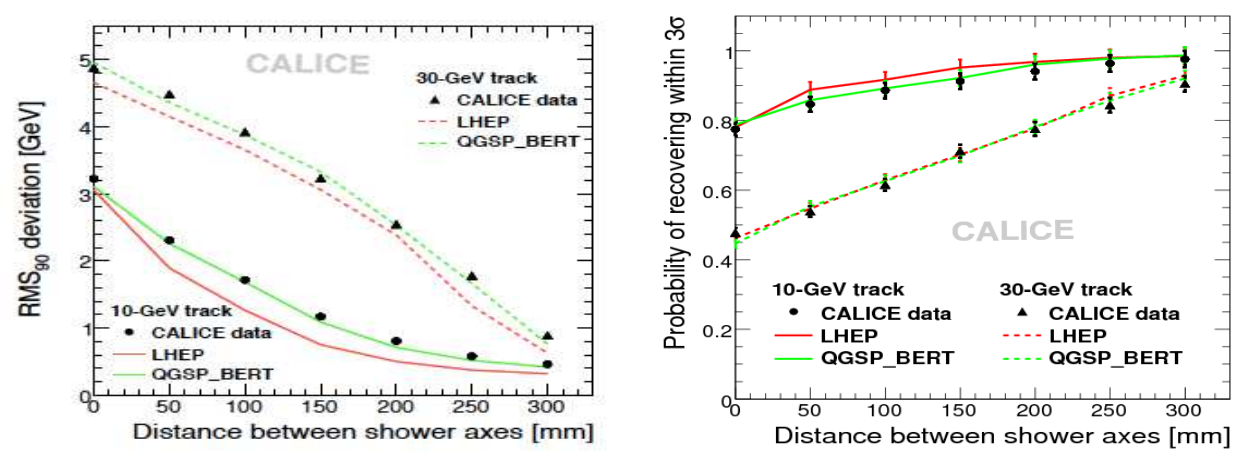

Figure 3: Confusion error (left) and a probability for correct (within $3 \sigma$ ) reconstruction (right) for a $10 \mathrm{GeV}$ neutral shower in the vicinity of $10 \mathrm{GeV}$ (points) and $30 \mathrm{GeV}$ (triangles) charged showers. Lines show MC predictions.

track from one shower was removed to simulate a neutral shower. The PandoraPFA program[10] was used to reconstruct the energy of this "neutral" shower in the vicinity of the charged shower. The difference between reconstructed and measured energy of the "neutral" shower appears due to wrong assignment of hits to showers. This confusion term is the dominant source of the error in the jet energy reconstruction in the PFA approach. The confusion error is shown in Fig.3. In spite of a large values of the confusion error for small distances between showers the PandoraPFA is often capable to reconstruct correctly (within $3 \sigma$ ) the "neutral" shower energy even at zero distances between showers (see Fig.3). The agreement between the PandoraPFA performance with real calorimeter prototype data and with the MC simulation demonstrates that the extrapolation to the complete detector is reliable. No hidden imperfections in the real data (imperfect calibration, non-uniformity of tile response, cross talk between tiles, dead or noisy channels) which could degrade the PFA performance were found. Thus the most critical part of the PFA approach passed the experimental tests.

\section{References}

[1] J.Repond et al. (CALICE Collaboration), JINST 3 (2008) P08001.

[2] C.Adloff et al. (CALICE Collaboration), JINST 5 (2010) P05004 [arXiv:1003.2662].

[3] Mokka home page, http://polzope.in2p3.fr:8081/MOKKA.

[4] C.Adloff et al. (CALICE Collaboration), JINST 5(2010) P05007 [arXiv:1004.4996].

[5] A. Kaplan, Pion Showers in the CALICE AHCAL Prototype, CALICE Analysis Note 26.

[6] A.Kaplan, in proceeding of 2nd Int.Conf. on Technology and Instrumentation in Particle Physics.

[7] F.Simon, in proceeding of 2nd Int.Conf. on Technology and Instrumentation in Particle Physics.

[8] M.Chadeeva, K.Seidel, CALICE Analysis Note 35.

[9] C.Adloff et al. (CALICE Collaboration), JINST 6(2011) P07005[arXiv:1105.3417].

[10] M.A.Thomson, NIM A611 (2009) 25. 